\title{
Construction of Optimal Portfolio Using Efficient Portfolio and Zero Opportunity Cost
}

\author{
Daniel Arficho Gaenore ${ }^{1 *}$, M Montaz Ali $^{2}$, Aduda Jane Akinyi ${ }^{3}$ \\ ${ }^{1}$ Department of Statistics and Actuarial Sciences, Faculty of mathematics, Pan African University for Basic Sciences, \\ Technology and Innovations, Nairobi, Kenya \\ ${ }^{2}$ School of Computer Science and Applied Mathematics, University of the Witwatersrand, Johannesburg, South Africa \\ ${ }^{3}$ Department of Statistics and Actuarial Sciences, Faculty of mathematics, JKUAT, Nairobi, Kenya
}

Cite This Paper in the following Citation Styles

(a): [1] Daniel Arficho Gaenore, M Montaz Ali, Aduda Jane Akinyi, "Construction of Optimal Portfolio Using Efficient Portfolio and Zero Opportunity Cost," Universal Journal of Accounting and Finance, Vol.10, No.2, pp. 609-623, 2022. DOI: 10.13189/ujaf.2022.100226

(b): Daniel Arficho Gaenore, M Montaz Ali, Aduda Jane Akinyi, (2022). Construction of Optimal Portfolio Using Efficient Portfolio and Zero Opportunity Cost. Universal Journal of Accounting and Finance, 10(2), 609-623. DOI: 10.13189/ujaf.2022.100226

Copyright $\odot 2022$ by authors, all rights reserved. Authors agree that this article remains permanently open access under the terms of the Creative Commons Attribution License 4.0 International License

\begin{abstract}
In this manuscript, we introduce asset allocation and portfolio selection techniques based on efficiency condition, Sharpe ratio error condition and order three zero opportunity condition. Investors expect the same level of risk and return from alternative investment options unless they want the advantage of diversification for risk. There are two fundamental investment portfolios. The first one is risk free fundamental portfolio, and the second one is risky fundamental portfolio. Investors use zero opportunity cost to select portfolio objective. In this research, mathematical derivation of portfolio construction approach is described in advance. Historical data of this research show that there is a positive linear relationship between natural logarithm of standard deviation of securities' return and square of co-variance between securities and market return. Furthermore, it shows that there is a positive linear relationship between Treynor ratio of securities' return and Sharpe ratio of securities' return. We use global search optimization tool in MatLab and R software to solve empirical portfolio selection and asset allocation problem. Moreover, we apply direct and indirect mathematical proof methods to prove mathematical facts of this study.
\end{abstract}

Keywords Asset, Efficient Frontier, Opportunity Cost, Return Neutral, Sharpe Ratio

\section{Introduction}

Investors optimize investment asset allocation to reduce unnecessary losses based on their investment objectives and constraints. Traditionally, investors use 40-60 asset allocation strategies. This 40-60 classical asset allocation strategy need to be optimal. Thus, investors should set their investment objectives and constraints to optimize asset allocation. Practitioners use mean variance model to select portfolio even if the model assumes weights of securities are independent of time parameter. Another problem of mean variance model is that the model does not consider zero opportunity cost between fundamental portfolios. Single index model is developed because mean variance model needs estimations of expected return, risk and covariance of securities. This indicates that mean variance model is not easy for portfolio with large number of securities. In this research, we propose new portfolio construction method using order three zero opportunity condition, efficiency condition and Sharpe ratio error condition. Clearly, one can express variance of portfolio in terms of expected return by using efficiency condition. Similarly, the variance of portfolio can be expressed in terms of expected return due to Sharpe ratio error condition. Let $w_{f}^{*}, w_{p}^{*}$ and $w_{m}^{*}$ be weight of risk free security, weight of risky portfolio and weight of market portfolio, respectively. Suppose that $r_{f}, r_{t p}$ and $r_{t m}$ represents risk free rate of return, risky portfolio rate of return and market portfolio rate of return at time $t$, respectively. Then we define rate of return of complete portfolio $r_{t c p}$ by $r_{t c p}=w_{f}^{*} r_{f}+w_{p}^{*} r_{t p}+w_{m}^{*} r_{t m}$. In this research, we determine $r_{t p}, w_{f}^{*}, w_{p}^{*}$ and $w_{m}^{*}$ using order three zero opportunity condition, efficiency condition and Sharpe ratio error condition. Moreover, we use weight-variance regres- 
sion model and Treynor-Sharpe regression model to build General order three opportunity model (GOOM). Furthermore, we solve Order three asset allocation model (OAAM) to determine complete portfolio return. We use USA $s \& p 500$ companies one year daily returns for empirical study.

\subsection{Motivation}

Since investors have different objectives, their portfolio selection need not be the same using mean variance model. So which investor is right? This question motivates researchers to study portfolio selection and asset allocation techniques using zero opportunity cost.

\subsection{Statement of the problem}

The statement of the problem is constructing optimal complete portfolio using order three zero opportunity condition, efficiency condition and Sharpe ratio error condition. Mathematically, we obtain the optimal risk free security weight $w_{f}^{*}$, market portfolio weight $w_{m}^{*}$ and risky portfolio weight $w_{p}^{*}$ to construct complete portfolio $P_{c}$ such that $r_{t c p}=w_{f}^{*} r_{f}+w_{p}^{*} r_{t p}+$ $w_{m}^{*} r_{t m}$ using order three zero opportunity condition, efficiency condition and Sharpe ratio error condition.

\subsection{Objectives of the study}

The general objective of this study is to construct complete portfolio using order three zero opportunity condition, efficiency condition and Sharpe ratio error condition. Specific objectives are:

1. to show that there is positive linear relation ship between Treynor ratio and Sharpe ratio of securities,

2. to estimate parameters of weight-variance regression model.

3. to build General order three opportunity model (GOOM),

4. to construct Order three asset allocation model (OAAM).

\subsection{Research gap}

Since efficient frontier contains many efficient portfolios, investors should choose efficient portfolio using logical constraints. Mean variance model does not explain how to choose efficient portfolio using logical constraints. In this research, we use (GOOM) and (OAAM) to construct optimal complete portfolio.

\section{Literature review}

Investment is an allocation of resources for medium or long term and the expected effect is to recover the investment costs and have a high profit, [1]. Risk is the possibility that desired objective would not be attained, [2]. One cannot definitely determine whether the risk is only good or bad, [3].

\subsection{Financial risk preference and tolerance}

Risk preference is the individual's orientation towards risktaking, [4]. Financial risk tolerance is the level of risk that clients believe they are willing to accept, [5]. Financial risk tolerance is the maximum amount of volatility that investor is willing to accept when making a financial decision, [5]. Risk aversion means an attitude of reluctance to take risky decisions, [6]. Financial Risk Tolerance is the willingness of individual investors to receive negative changes of investment value or the result opposite or different from the expected results, [7]. Willingness to take financial risk depends on portfolio structure, gender, age, educational attainment, income, financial stability, financial literacy, marital status, and family size, [8].

Define standard deviation vector $\sigma$, weight vector $w$, expected return vector $\mu$ and vector of ones $l$ as follows. $\sigma=\left(\sigma_{1}, \sigma_{2}, \ldots, \sigma_{n}\right)^{T}, w=\left(w_{1}, w_{2}, \ldots, w_{n}\right)^{T}, \mu=$ $\left(\mu_{1}, \mu_{2}, \ldots, \mu_{n}\right)^{T}$ and $l=(1,1, \ldots, 1)^{T}$. Let $\Sigma$ be covariance matrix of order $n$.

\subsection{Markowitz' mean variance model}

H.M. Markowitz' was the first who introduced Markowitz' mean variance model for portfolio selection.

The Markowitz' model $(M M)$ is given by:

$$
\begin{aligned}
M M & \max _{w} \mu^{T} w \\
& \min _{w} w^{T} \Sigma w, \\
\text { subject to } & l^{T} w=1 .
\end{aligned}
$$

Note that return maximization and risk minimization objectives are conflicting objectives. If investors attempt to increase return, they will be penalized by risk. The solution of $M M$ depends on investors' risk preference. Consider the following special cases of $M M$.

\subsection{Risk neutral investors}

Risk neutral investors are investors who are interested to maximize return without paying attention on risk, [9]. These investors do not care about risk minimization. Risk neutral Markowitz's model (RINMM) is given as follows:

$$
\begin{array}{ll}
R I N M M & \max _{w} \mu^{T} w \\
\text { subject to } & l^{T} w=1 .
\end{array}
$$

\subsection{Return neural investors}

Some investors prefer risk minimization rather than return maximization. These investors are called return neutral investors. Return neutral Markowitz's model is given as follows:

$$
\begin{aligned}
R E N M M & \min _{w} w^{T} \Sigma w, \\
\text { subject to } & l^{T} w=1 .
\end{aligned}
$$

Note that return neutral investors gain less return than risk neutral investors. However, they are less exposed to risk. 


\subsection{Maximum expected return for a given level of risk}

Investors who know their risk tolerance potential would like to maximize return for their risk level. These investors would like to solve the following optimization problem:

$$
\begin{array}{ll} 
& \max _{w} \mu^{T} w \\
\text { subject to } & w^{T} \Sigma w=\sigma_{p}^{*}, \\
& l^{T} w=1 .
\end{array}
$$

Readers are referred to [9] for further detail about maximum expected return for a given level of risk.

\subsection{Minimum risk for a given level of expected return}

Those investors who are interested to achieve targeted expected return by minimizing risk use the following model:

$$
\begin{array}{ll} 
& \min _{w} w^{T} \Sigma w \\
\text { subject to } & \mu^{T} w=\mu_{p}^{*}, \\
& l^{T} w=1 .
\end{array}
$$

The detail of this model is given in [9].

\subsection{Risk adjusted model (RAM)}

Investors may have different weight levels for risk and return. The investors who think both risk and return are equally important use 50-50 weights for risk and return. In general, risk adjusted portfolio model has the following form as given in $[9,10]$ :

$$
\begin{aligned}
r A M & \min _{w}\left[\rho w^{T} \Sigma w-\mu^{T} w\right] \\
\text { subject to } & l^{T} w=1,
\end{aligned}
$$

where $\rho$ is a parameter.

\subsection{Sharpe ratio maximization model $(S R M M)$}

Portfolio comparison is relative to some reference. For instance, managers may compare two portfolios relative to Sharpe ratio. This does not mean portfolio with high Sharpe ratio is absolutely better than portfolios with low Sharpe ratio. However, portfolio with high Sharpe ratio is more desirable than the one with low Sharpe ratio provided an investor cares about Sharpe ratio rather than other criteria. See reference $[11,12]$ for more details. Sharpe ratio maximization model is given by:

$$
\begin{array}{ll}
\text { SRMM } & \max _{w} \frac{\mu^{T} w-r_{f}}{\sqrt{w^{T} \Sigma w}} \\
\text { subject to } & l^{T} w=1
\end{array}
$$

where $r_{f}$ risk free rate of return.

\subsection{Diversification maximization model $(D M M)$}

Diversification is a key technique to minimize investment risk. Investors who think diversification as a good tool to minimize risk maximize diversification ratio. Diversification ratio maximization model is given in, [13]. The following model is called diversification maximization model.

$$
\begin{aligned}
D M M & \max _{w} \frac{\sigma^{T} w}{\sqrt{w^{T} \Sigma w}} \\
\text { subject to } & l^{T} w=1 .
\end{aligned}
$$

The above investment portfolio construction models presented above are well known fundamental portfolio selection models. However, there are some issues which also affect portfolio selection decision. For instance, managers should consider opportunity cost, variability (instability) of security weight over time, inefficiency, return errors and market factors when they construct portfolio. In this research, portfolio errors such as return error, frontier error, opportunity error, Sharpe error, Treynor error, Sharpe-Treynor error and weight error are considered.

\subsection{Global minimum variance and expected return}

Suppose that investors want to maximize expected return for a given level of risk, see problem (4)-(5). Apply Lagrange multiplier method to solve expected return maximization problem for a given risk level. Let $\lambda_{1}$ and $\lambda_{2}$ be two scalars. Then define

$$
L\left(w, \lambda_{1}, \lambda_{2}\right)=-\mu^{T} w-\lambda_{2}\left(l^{T} w-1\right)-\lambda_{1}\left(w^{T} \Sigma w-\sigma_{p}^{*}\right) .
$$

Solve $\nabla L\left(w, \lambda_{1}, \lambda_{2}\right)=0$ to find critical points of $L$. Thus, the following equations follow from $\nabla L\left(w, \lambda_{1}, \lambda_{2}\right)=0$.

$$
\begin{aligned}
& \Sigma w=\frac{-\mu-\lambda_{2} l}{2 \lambda_{1}}, \\
& l^{T} w=1, \\
& w^{T} \Sigma w=\sigma_{p}^{*} .
\end{aligned}
$$

This implies that

$$
\begin{aligned}
& w^{T} \Sigma=\frac{-\mu^{T}-\lambda_{2} l^{T}}{2 \lambda_{1}}, \\
& l^{T} w=1, \\
& w^{T} \Sigma w=\sigma_{p}^{*} .
\end{aligned}
$$

It follows from equation (14) that

$$
w^{T}=\frac{-\mu^{T} \Sigma^{-1}-\lambda_{2} l^{T} \Sigma^{-1}}{2 \lambda_{1}} .
$$

This implies that

$$
\left(w^{T} l\right) 2 \lambda_{1}=-\mu^{T} \Sigma^{-1} l-\lambda_{2} l^{T} \Sigma^{-1} l .
$$

Since $w^{T} l=1$, we get

$$
2 \lambda_{1}=-b-\lambda_{2} c .
$$

Thus, the following equations are valid. 


$$
\begin{gathered}
\lambda_{2}=\frac{-\left(b+2 \lambda_{1}\right)}{c}, \\
\sigma_{p}^{*}=\left(\frac{1}{2 \lambda_{1}}\right)^{2}\left(\frac{a c-b^{2}}{c}\right)+\frac{1}{c} .
\end{gathered}
$$

It follows from equation (17) that

$$
\mu_{p}=w^{T} \mu=\frac{-\mu^{T} \Sigma^{-1} \mu-\lambda_{2} l^{T} \Sigma^{-1} \mu}{2 \lambda_{1}}=\frac{-a-\lambda_{2} b}{2 \lambda_{1}} .
$$

Substitute the value of $\lambda_{2}$ in to the equation (22) to get the following equation (23).

$$
\frac{1}{2 \lambda_{1}}=\frac{b-c \mu_{p}}{a c-b^{2}}
$$

Substitute equation (23) in to the equation (21) to get constant weight efficient frontier of portfolio $p$. Therefore, constant weight efficient frontier of portfolio $p$ is given by:

$$
\sigma_{p}^{2}=\frac{1}{c}\left(1+\frac{\left(c \mu_{p}-b\right)^{2}}{a c-b^{2}}\right) .
$$

Minimize $\sigma_{p}^{2}$ with respect to $\mu_{p}$ to derive global minimum variance of portfolio $p$.

The global minimum variance of portfolio $p$ is given by:

$$
\left(\sigma_{p}^{2}\right)^{*}=\frac{1}{c} .
$$

Clearly, the global minimum variance of portfolio $p$ is obtained at $\mu_{p}=\frac{b}{c}$, [14, 15, 16, 17].

\subsection{Complete portfolio}

A complete portfolio of risk free asset and risky asset portfolio is defined as a combination of a risky asset portfolio and the risk-free asset, [18]. The return $r_{c}$ of complete portfolio is defined by:

$$
r_{c}=w_{p} r_{p}+\left(1-w_{p}\right) r_{f},
$$

where $r_{p}$ is risky asset portfolio return, $r_{f}$ is risk free asset return and $w_{p}$ is wight of risky asset portfolio.

\subsection{Capital market line}

Capital Market Line (CML) contains all optimal portfolios that are combination partners of the risk-free asset and the market portfolio, [19]. All of the portfolios on the CML have the same Sharpe ratio as that of the market portfolio, [19]. The tangency portfolio is the intersection point of the CML and the efficient frontier, $[17,20]$. Let $r_{m}$ be return of market portfolio. Then the return of tangency portfolio $r_{p}$ is defined by:

$$
r_{p}=w_{p} r_{m}+\left(1-w_{p}\right) r_{f},
$$

where $r_{f}$ is risk free asset return and $w_{p}$ is wight of market portfolio. In this research, we relax the tangency portfolio condition $r_{p}=w_{p} r_{m}+\left(1-w_{p}\right) r_{f}$ to order two opportunity cost condition $\rho r_{p}+(1-\rho) r_{m}=w_{p} r_{m}+\left(1-w_{p}\right) r_{f}$, where $\rho \in[0,1]$. Note that tangency portfolio condition is a special case of order two opportunity cost condition, choose $\rho=1$.

We observe from literature review that portfolio selection models depend on investors' decisions and objectives. Tangency portfolio condition helps investors to choose efficient portfolio when investors would like to construct a complete portfolio of risk free asset and market index. However, there exist order two and three zero opportunity condition which are general conditions of Tangency portfolio condition. In summary, researchers study portfolio selection techniques based on zero opportunity cost condition as investors' decision.

\section{Portfolio selection using zero opportunity cost}

This research presents the mathematical modeling for portfolio selection based on zero opportunity cost. Hence there are many mathematical expressions used throughout this research. We have used a number symbols in this mathematical expressions which are given in Table 1.

\begin{tabular}{|c|l|}
\hline Symbol & Value \\
\hline$a$ & $\mu^{T} \Sigma^{-1} \mu$ \\
\hline$b$ & $\mu^{T} \Sigma^{-1} l$ \\
\hline$c$ & $l^{T} \Sigma^{-1} l$ \\
\hline$z$ & $\left(\frac{\sigma_{m}}{\mu_{m}-r_{f}}\right)^{2}$ \\
\hline$x$ & $\frac{-w_{p} r_{f}+\left(w_{p}-1\right) \mu_{m}}{\mu_{m}-r_{f}}$ \\
\hline$x^{*}$ & $\left(1-w_{p}\right)+\frac{r_{f} \mu_{m}}{\left(\mu_{m}-r_{f}\right)^{2}}$ \\
\hline$s^{*}$ & $\sigma_{m}^{2}\left(x^{*}+x\right)$ \\
\hline$q^{*}$ & $\frac{-2 \sigma_{m}^{2} r_{f}}{\left(\mu_{m}-r_{f}\right)^{2}}$ \\
\hline
\end{tabular}

Table 1. Symbols and their corresponding value

\subsection{Frontier error minimization model $(F E M M)$}

The following model is called frontier error minimization model:

$$
\text { FEMM }: \min _{w}\left(w^{T} \Sigma w-\frac{1}{c}\left(1+\frac{\left(c \mu^{T} w-b\right)^{2}}{a c-b^{2}}\right)\right)^{2}
$$

Subject to : $l^{T} w=1$.

A portfolio that corresponds to $F E M M$ is called frontier error $(F E)$ portfolio.

Definition 3.2 (Efficiency condition). The equation

$$
w^{T} \Sigma w=\frac{1}{c}\left(1+\frac{\left(c \mu^{T} w-b\right)^{2}}{a c-b^{2}}\right)
$$

is called efficiency condition.

\subsection{Zero opportunity portfolio}

Let $r_{f}$ be the rate of return of risk free asset. Let $r_{m}$ be return of market portfolio (index). Let $\mu_{m}$ be expected return of market portfolio. Let $r_{p}$ be return of risky portfolio. Scenario 
one portfolio (risk free fundamental portfolio) $S P_{1}$ is a portfolio with return $r_{S P_{1}}=w_{p} r_{f}+\left(1-w_{p}\right) r_{m}, w_{p} \in[0,1]$. Scenario two portfolio (risky fundamental portfolio) $S P_{2}$ is a portfolio with return $r_{S P_{2}}=\rho r_{p}+(1-\rho) r_{m}, \rho \in[0,1]$. Scenario three portfolio (free-risky fundamental portfolio) $S P_{3}$ is a portfolio with return $r_{S P_{3}}=\delta r_{f}+(1-\delta) r_{p}, \delta \in[0,1] . S P_{1}$ and $S P_{2}$ are said to be zero opportunity portfolios if $\mu_{m} \neq r_{f}$, $w_{p} \neq 0, \rho=w_{p}$ and $r_{S P_{1}}=r_{S P_{2}}$.

$S P_{1}$ and $S P_{2}$ are said to be equivalent portfolios of order two if $r_{S P_{1}}=r_{S P_{2}}$. $S P_{1}, S P_{2}$ and $S P_{3}$ are said to be equivalent portfolios of order three if $r_{S P_{1}}=r_{S P_{2}}=r_{S P_{3}}$. Consider equivalent portfolios of order two. There are two cases for equivalent portfolios of order two. The first case $\rho=w_{p}$. The second case for equivalent portfolios of order two is $\rho \neq w_{p}$. Let us begin with $\rho=w_{p}$ case. The following results are obtained by the help of $\rho=w_{p}$ case.

Theorem 3.4. Let $\alpha_{i}$ and $\beta_{i}$ be two real numbers. Define

1. $\overline{r_{S P_{j}}}=E\left(r_{S P_{j}}\right)$ for $j=1,2,3$, where $E$ is expectation,

2. $\sigma_{S P_{j}}=S D\left(r_{S P_{j}}\right)$ for $j=1,2,3$, where $S D$ is standard deviation,

3. $\sigma_{j}=S D\left(r_{j}\right)$, where $S D$ is standard deviation,

4. $\rho_{p m}=\operatorname{corr}\left(r_{p}, r_{m}\right)$, where corr is correlation,

5. $h\left(w_{p}\right)=\sigma_{m}+\alpha_{p} w_{p}$,

6. $g\left(w_{p}\right)=\beta_{p}+\left(2 \alpha_{p} \sigma_{m}+2 \sigma_{m}^{2}\right) w_{p}+\left(\alpha_{p}^{2}-\sigma_{m}^{2}\right) w_{p}^{2}$.

If $S P_{1}$ and $S P_{2}$ are zero opportunity portfolios, then $2 \rho_{i m} w_{p}\left(1-w_{p}\right) \sigma_{m} \sigma_{p}=-w_{p}^{2} \sigma_{p}^{2}+g\left(w_{p}\right)$.

Proof

Suppose that $S P_{1}$ and $S P_{2}$ are zero opportunity portfolios.

Then

$$
\begin{gathered}
r_{S P_{1}}=w_{p} r_{f}+\left(1-w_{p}\right) r_{m}, \\
r_{S P_{2}}=\rho r_{p}+(1-\rho) r_{m}
\end{gathered}
$$

and

$$
w_{p} r_{f}+\left(1-w_{p}\right) r_{m}=\rho r_{p}+(1-\rho) r_{m} .
$$

It follows from equation (32) that

$$
\overline{r_{S P_{1}}}=\overline{r_{S P_{2}}}
$$

and

$$
\sigma_{S P_{1}}=\sigma_{S P_{2}}
$$

It follows from equation (30) that

$$
\sigma_{S P_{1}}=\left(1-w_{p}\right) \sigma_{m}
$$

Solve for $w_{p}$ from equation (35) to get equation (36).

$$
w_{p}=\frac{\sigma_{m}-\sigma_{S P_{1}}}{\sigma_{m}} .
$$

Clearly the following equations hold.

$$
\overline{r_{S P_{1}}}=w_{p} r_{f}+\left(1-w_{p}\right) \mu_{m} .
$$

$$
\overline{r_{S P_{2}}}=w_{p} \mu_{p}+\left(1-w_{p}\right) \mu_{m} .
$$

Substitute equation (36) in to the equation (37) to get (39).

$$
\overline{r_{S P_{1}}}=r_{f}+\left(\mu_{m}-r_{f}\right) \frac{\sigma_{S P_{1}}}{\sigma_{m}},
$$

Use equations (33), (38) and (39) to get equation (40).

$$
r_{f}+\left(\mu_{m}-r_{f}\right) \frac{\sigma_{S P_{1}}}{\sigma_{m}}=w_{p} \mu_{p}+\left(1-w_{p}\right) \mu_{m}
$$

It follows from equation (40) that

$$
\mu_{p}=\frac{r_{f}+\left(\mu_{m}-r_{f}\right) \frac{\sigma_{S P_{1}}}{\sigma_{m}}+\left(w_{p}-1\right) \mu_{m}}{w_{p}} .
$$

It follows from equations (39) and (40) that

$$
\overline{r_{S P_{1}}}=w_{p} \mu_{p}+\left(1-w_{p}\right) \mu_{m} .
$$

Note that market portfolio expected return and standard deviation are constant numbers, whereas weight $w_{p}$ and $\sigma_{p}$ are variables. Clearly,

$$
\sigma_{S P_{2}}=\sqrt{w_{p}^{2} \sigma_{p}^{2}+\left(w_{p}-1\right)^{2} \sigma_{m}^{2}+2 w_{p}\left(1-w_{p}\right) \rho_{p m} \sigma_{p} \sigma_{m}}
$$

Since $\mu_{p}=r_{f}, \frac{\partial \mu_{p}}{\partial w_{p}}=0$ and $\frac{\partial \mu_{p}}{\partial \sigma_{p}}=0$. Use $\sigma_{S P_{1}}=\sigma_{S P_{2}}$ and equation (43) to find equation (44).

$$
\sigma_{S P_{1}}=\sqrt{w_{p}^{2} \sigma_{p}^{2}+\left(w_{p}-1\right)^{2} \sigma_{m}^{2}+2 w_{p}\left(1-w_{p}\right) \rho_{p m} \sigma_{p} \sigma_{m}} .
$$

Solve for $w_{p}$ from equation (37) to get equation (45)

$$
w_{p}=\frac{\overline{r_{S P_{1}}}-\mu_{m}}{r_{f}-\mu_{m}} .
$$

Substitute equation (42) in to the equation (45) to find equation (46).

$$
w_{p}=\frac{w_{p} \mu_{p}+\left(1-w_{p}\right) \mu_{m}-\mu_{m}}{r_{f}-\mu_{m}} .
$$

Substitute equation (46) in to the equation (35) to get equation (47).

$$
\sigma_{S P_{1}}=\frac{r_{f}-w_{p} \mu_{p}+\left(w_{p}-1\right) \mu_{m}}{r_{f}-\mu_{m}} \sigma_{m} .
$$

Not that $\frac{\partial\left(w_{p} \mu_{p}\right)}{\partial w_{p}}=\frac{\partial\left(w_{p} r_{f}\right)}{\partial w_{p}}=r_{f}=\mu_{p}$.

Differentiate both sides of equation (47) with respect to $w_{p}$ to get equation (48).

$$
\begin{gathered}
\mu_{p}=\left(\mu_{m}-r_{f}\right) \frac{\frac{\partial \sigma_{S P_{1}}}{\partial w_{p}}}{\sigma_{m}}+\mu_{m} . \\
\frac{\partial \sigma_{S P_{1}}}{\partial \sigma_{p}}=0 .
\end{gathered}
$$

It follows from equation (49) that

$$
\sigma_{S P_{1}}=h\left(w_{p}\right)
$$


It follows from equations (41), (48) and (50) that

$$
\frac{h\left(w_{p}\right)}{\sigma_{m}}-w_{p} \frac{\frac{d h\left(w_{p}\right)}{d w_{p}}}{\sigma_{m}}=1 .
$$

We also note the following equations:

$$
\begin{gathered}
2 w_{p}\left(1-w_{p}\right) \rho_{i m} \sigma_{p} \sigma_{m}=-w_{p}^{2} \sigma_{p}^{2}+g\left(w_{p}\right) \\
\frac{d h\left(w_{p}\right)}{d w_{p}}=\frac{1}{2 h\left(w_{p}\right)}\left(2 w_{p} \sigma_{p}^{2}+2\left(w_{p}-1\right) \sigma_{m}^{2}+u\right),
\end{gathered}
$$

where $u=\frac{\partial\left(2 w_{p}\left(1-w_{p}\right) \rho_{p m} \sigma_{p} \sigma_{m}\right)}{\partial w_{p}}$. It follows from equations (52) and (53) that

$$
\frac{d g\left(w_{p}\right)}{d w_{p}}=2 h\left(w_{p}\right) \frac{d h\left(w_{p}\right)}{d w_{p}}+2\left(1-w_{p}\right) \sigma_{m}^{2} .
$$

Solve for $h$ from equation (51). Substitute $h$ in to equation (54) and solve for $g$ from equation (54). Hence proved.

Theorem 3.5. If $S P_{1}$ and $S P_{2}$ are zero opportunity portfolios, then

$$
\begin{aligned}
& \text { 1. } \alpha_{p}=\frac{\mu_{p}-\mu_{m}}{\mu_{m}-r_{f}} \sigma_{m}, \\
& \text { 2. } g\left(w_{p}\right)=\left(2 \alpha_{p} \sigma_{m}+2 \sigma_{m}^{2}\right) w_{p}+\left(\alpha_{p}^{2}-\sigma_{m}^{2}\right) w_{p}^{2}, \\
& \text { 3. } 2 \rho_{p m} w_{p}\left(1-w_{p}\right) \sigma_{m} \sigma_{p}=-w_{p}^{2} \sigma_{p}^{2}+g\left(w_{p}\right) .
\end{aligned}
$$

Proof. Clearly, $\alpha_{p}$ result follows from equation (48). Now solve for $\sigma_{S P_{1}}$ from equation (41). Thus, the following result follows:

$$
\sigma_{S P_{1}}=\sigma_{m}+w_{p} \alpha_{p}
$$

It follows from theorem 3.4 that

$$
\sigma_{S P_{1}}^{2}=\left(1-w_{p}\right)^{2} \sigma_{m}^{2}+g\left(w_{p}\right)
$$

The value of $\beta$ is zero from equations (55) and (56). Hence the proof followed.

Lemma 3.6. If risky and risk free fundamental portfolios have equal Sharpe ratio, then

$$
\sigma_{S P_{2}}=\frac{\overline{r_{S P_{2}}}-r_{f}}{\mu_{m}-r_{f}} \sigma_{m} .
$$

Proof. Suppose that risky and risk free fundamental portfolios have equal Sharpe ratio. Clearly,

$$
w_{p}=\frac{\overline{r_{S P_{2}}}-\mu_{m}}{\mu_{p}-\mu_{m}} .
$$

Since both risky and risk free fundamental portfolios have equal Sharpe ratio, we get

$$
\frac{\mu_{m}-r_{f}}{\sigma_{m}} \sigma_{S P_{2}}=w_{p}\left(\mu_{p}-\mu_{m}\right)+\mu_{m}-r_{f}
$$

Solve for $w_{p}$ from equation (59). Thus, the following equation follows.

$$
w_{p}=\frac{\left(\sigma_{S P_{2}}-\sigma_{m}\right)\left(\mu_{m}-r_{f}\right)}{\left(\mu_{p}-\mu_{m}\right) \sigma_{m}} .
$$

Use equations (58) and (60) to get equation (61).

$$
\frac{\left(\sigma_{S P_{2}}-\sigma_{m}\right)\left(\mu_{m}-r_{f}\right)}{\left(\mu_{p}-\mu_{m}\right) \sigma_{m}}=\frac{\overline{r_{S P_{2}}}-\mu_{m}}{\mu_{p}-\mu_{m}} .
$$

It follows from equation (61) that

$$
\sigma_{S P_{2}}=\frac{\overline{r_{S P_{2}}}-r_{f}}{\mu_{m}-r_{f}} \sigma_{m}
$$

Hence the proof followed.

Corollary 3.7. If $S P_{1}$ and $S P_{2}$ are zero opportunity portfolios, then

$$
\begin{aligned}
& \text { 1. } \alpha_{p}=\frac{\mu_{p}-\mu_{m}}{\mu_{m}-r_{f}} \sigma_{m}, \\
& \text { 2. } g\left(w_{p}\right)=\left(2 \alpha_{p} \sigma_{m}+2 \sigma_{m}^{2}\right) w_{p}+\left(\alpha_{p}^{2}-\sigma_{m}^{2}\right) w_{p}^{2}, \\
& \text { 3. } \sigma_{S P_{2}}^{2}=\left(1-w_{p}\right)^{2} \sigma_{m}^{2}+g\left(w_{p}\right) .
\end{aligned}
$$

Proof. The result of this theorem follows from Theorem 3.5.

Theorem 3.8. If $S P_{1}$ and $S P_{2}$ are zero opportunity portfolios, then

$$
\sigma_{S P_{2}}^{2}=z{\overline{r_{S P_{2}}}}^{2}+q^{*} \overline{r_{S P_{2}}}+s^{*} .
$$

Proof. Suppose that $S P_{1}$ and $S P_{2}$ are zero opportunity portfolios. Then

$$
\overline{r_{S P_{2}}}=w_{p} \mu_{p}+\left(1-w_{p}\right) \mu_{m}
$$

This implies that

$$
\mu_{p}=\frac{\overline{r_{S P_{2}}}+\left(w_{p}-1\right) \mu_{m}}{w_{p}} .
$$

Clearly,

$$
\alpha_{p}=\frac{\mu_{p}-\mu_{m}}{\mu_{m}-r_{f}} \sigma_{m} .
$$

It follows from equations (65) and (66) that

$$
\alpha_{p}=\frac{\sigma_{m}}{w_{p}}\left(\frac{\overline{r_{S P_{2}}}-\mu_{m}}{\mu_{m}-r_{f}}\right) .
$$

Consider the following equation.

$$
\begin{aligned}
\left(2 \alpha_{p} \sigma_{m}+2 \sigma_{m}^{2}\right) w_{p} & =\left(2 \frac{\sigma_{m}}{w_{p}}\left(\frac{\overline{r_{S P_{2}}}-\mu_{m}}{\mu_{m}-r_{f}}\right) \sigma_{m}+2 \sigma_{m}^{2}\right) w_{p} \\
& =2 \sigma_{m}\left(\frac{\overline{r_{S P_{2}}}-\mu_{m}}{\mu_{m}-r_{f}}\right) \sigma_{m}+2 \sigma_{m}^{2} w_{p} \\
& =2 \sigma_{m}^{2}\left(\left(\frac{\overline{r_{S P_{2}}}-\mu_{m}}{\mu_{m}-r_{f}}\right)+w_{p}\right) \\
& =2 \sigma_{m}^{2}\left(\frac{\overline{r_{S P_{2}}}+\left(w_{p}-1\right) \mu_{m}-w_{p} r_{f}}{\mu_{m}-r_{f}}\right) \\
& =2 \sigma_{m}^{2}\left(\frac{\overline{r_{S P_{2}}}}{\mu_{m}-r_{f}}+x\right) \\
& =\left(2 \mu_{m}-2 r_{f}\right)\left(\frac{\sigma_{m}}{\mu_{m}-r_{f}}\right)^{2} \overline{r_{S P_{2}}} \\
& +2 \sigma_{m}^{2} x .
\end{aligned}
$$


Let us consider the following equation.

$$
\begin{aligned}
\left(\alpha_{p}^{2}-\sigma_{m}^{2}\right) w_{p}^{2} & =\left(\frac{\sigma_{m}^{2}}{w_{p}^{2}}\left(\frac{\overline{r_{S P_{2}}}-\mu_{m}}{\mu_{m}-r_{f}}\right)^{2}-\sigma_{m}^{2}\right) w_{p}^{2} \\
& =\sigma_{m}^{2}\left(\frac{\overline{r_{S P_{2}}}-\mu_{m}}{\mu_{m}-r_{f}}\right)^{2}-\sigma_{m}^{2} w_{p}^{2} \\
& =\left(\frac{\sigma_{m}}{\mu_{m}-r_{f}}\right)^{2}\left(\overline{r_{S P_{2}}}-\mu_{m}\right)^{2}-\sigma_{m}^{2} w_{p}^{2} \\
& =\left(\frac{\sigma_{m}}{\mu_{m}-r_{f}}\right)^{2}\left({\overline{r_{S P_{2}}}}^{2}-2 \mu_{m} \overline{r_{S P_{2}}}+\mu_{m}^{2}\right) \\
& -\sigma_{m}^{2} w_{p}^{2} .
\end{aligned}
$$

It follows from corollary 3.7 that

$$
\sigma_{S P_{2}}^{2}=\left(1-w_{p}\right)^{2} \sigma_{m}^{2}+g\left(w_{p}\right) .
$$

Substitute equations (68) and (69) in to the equation (70) to get (71).

$$
\begin{aligned}
& \sigma_{S P_{2}}^{2}=\left(1-w_{p}\right)^{2} \sigma_{m}^{2}+-2 r_{f}\left(\frac{\sigma_{m}}{\mu_{m}-r_{f}}\right)^{2} \overline{r_{S P_{2}}}+2 \sigma_{m}^{2} x+ \\
&\left(\frac{\sigma_{m}}{\mu_{m}-r_{f}}\right)^{2}\left({\overline{r_{S P_{2}}}}^{2}+\mu_{m}^{2}\right)-\sigma_{m}^{2} w_{p}^{2}
\end{aligned}
$$

Define $d_{u}=\sigma_{m}^{2} x+\left(\frac{\sigma_{m}}{\mu_{m}-r_{f}}\right)^{2} \mu_{m}^{2}-\sigma_{m}^{2} w_{p}^{2}$. Consider the following equation.

$$
\begin{aligned}
d_{u} & =\sigma_{m}^{2} \frac{\left(w_{p}-1\right) \mu_{m}-w_{p} r_{f}}{\mu_{m}-r_{f}} \\
& +\left(\frac{\sigma_{m}}{\mu_{m}-r_{f}}\right)^{2} \mu_{m}^{2}-\sigma_{m}^{2} w_{p}^{2}
\end{aligned}
$$

This implies that

$$
\begin{aligned}
d_{u} & =\sigma_{m}^{2} \frac{\left(w_{p}-1\right) \mu_{m}-w_{p} r_{f}}{\mu_{m}-r_{f}}+\left(\frac{\sigma_{m}}{\mu_{m}-r_{f}}\right)^{2} \mu_{m}^{2}-\sigma_{m}^{2} w_{p}^{2} \\
& =\sigma_{m}^{2}\left(\frac{\left(w_{p}-1\right) \mu_{m}-w_{p} r_{f}}{\mu_{m}-r_{f}}\right)+\sigma_{m}^{2}\left(\left(\frac{\mu_{m}}{\mu_{m}-r_{f}}\right)^{2}-w_{p}^{2}\right) \\
& =\sigma_{m}^{2}\left(\frac{\left(w_{p}-1\right) \mu_{m}-w_{p} r_{f}}{\mu_{m}-r_{f}}+\left(\frac{\mu_{m}}{\mu_{m}-r_{f}}-w_{p}\right)\left(\frac{\mu_{m}}{\mu_{m}-r_{f}}+w_{p}\right)\right. \\
& =\sigma_{m}^{2}\left(\frac{\left(w_{p}-1\right) \mu_{m}-w_{p} r_{f}}{\mu_{m}-r_{f}}\right)\left(1-\left(\frac{\mu_{m}}{\mu_{m}-r_{f}}+w_{p}\right)\right) \\
& =\sigma_{m}^{2}\left(\frac{\left(w_{p}-1\right) \mu_{m}-w_{p} r_{f}}{\mu_{m}-r_{f}}\right)\left(\frac{-w_{p} \mu_{m}-\left(1-w_{p}\right) r_{f}}{\mu_{m}-r_{f}}\right) \\
& =\sigma_{m}^{2}\left(\frac{\left(1-w_{p}\right) \mu_{m}+w_{p} r_{f}}{\mu_{m}-r_{f}}\right)\left(\frac{w_{p} \mu_{m}+\left(1-w_{p}\right) r_{f}}{\mu_{m}-r_{f}}\right) \\
& =\left(\frac{\sigma_{m}}{\mu_{m}-r_{f}}\right)^{2}\left(\left(1-w_{p}\right) \mu_{m}+w_{p} r_{f}\right)\left(w_{p} \mu_{m}+\left(1-w_{p}\right) r_{f}\right)
\end{aligned}
$$

Define $d_{l}=\left(\left(1-w_{p}\right) \mu_{m}+w_{p} r_{f}\right)\left(w_{p} \mu_{m}+\left(1-w_{p}\right) r_{f}\right)$. Note that

$$
\begin{aligned}
d_{l} & =\left(w_{p}-w_{p}^{2}\right)\left(r_{f}^{2}+\mu_{m}^{2}\right)+\left(1-2 w_{p}+2 w_{p}^{2}\right) r_{f} \mu_{m} \\
& =\left(w_{p}-w_{p}^{2}\right)\left(\mu_{m}-r_{f}\right)^{2}+r_{f} \mu_{m} .
\end{aligned}
$$
by:
It follows from equations (73) and (74) that

$$
d_{u}=\left(w_{p}-w_{p}^{2}\right) \sigma_{m}^{2}+r_{f} \mu_{m}\left(\frac{\sigma_{m}}{\mu_{m}-r_{f}}\right)^{2} .
$$

Substitute equation (75) in to the equation (71) to get equation (76).

$$
\sigma_{S P_{2}}^{2}=\left(1-w_{p}\right) \sigma_{m}^{2}+q^{*} \bar{r}_{S P_{2}}+\sigma_{m}^{2} x+z{\overline{r_{S P_{2}}}}^{2}+r_{f} \mu_{m}\left(\frac{\sigma_{m}}{\mu_{m}-r_{f}}\right)^{2} .
$$

This implies that

$$
\sigma_{S P_{2}}^{2}=z{\overline{r_{S P_{2}}}}^{2}+q^{*} \overline{r_{S P_{2}}}+s^{*} .
$$

Hence the proof followed.

\subsection{Sharpe square error model $(S S E M)$}

We define zero Sharpe square error $Z S S E$ by:

$$
Z S S E=\left(\sigma_{S P_{2}}-\frac{\overline{r_{S P_{2}}}-r_{f}}{\mu_{m}-r_{f}} \sigma_{m}\right)^{2}
$$

Investors who prefer to construct efficient portfolio using zero Sharpe square error would like to solve Sharpe square error model. Sharpe square error model is given by:

$$
S S E M: \min _{w_{p}, w}\left(\sigma_{S P_{2}}-\frac{\overline{r_{S P_{2}}}-r_{f}}{\mu_{m}-r_{f}} \sigma_{m}\right)^{2}
$$

Subject to : $l^{T} w=1$,

$$
0 \leq w_{p}, w_{i} \leq 1 \forall i=1,2, \ldots, n .
$$

A portfolio that corresponds to $S S E M$ is called Sharpe square error $(S S E)$ portfolio.

Definition 3.10 (Sharpe ratio error condition). The equation

$$
\sigma_{S P_{2}}=\frac{\overline{r_{S P_{2}}}-r_{f}}{\mu_{m}-r_{f}} \sigma_{m}
$$

is called Sharpe ratio error condition.

\subsection{Return neutral portfolio}

Use return neutral model to construct return neutral portfolio. Clearly, $\vec{w}=\frac{\Sigma^{-1} l}{c}$.

\subsection{Sharpe ratio efficient opportunity portfolio}

Sharpe ratio efficient opportunity model $(S R E O M)$ is an optimization model with Sharpe ratio objective subject to efficient-opportunity constraint and unit weight sum constraint. Sharpe ratio efficient opportunity model $(S R E O M)$ is given

$$
\begin{array}{ll}
\text { SREOM : } & \max _{w} \frac{\mu_{p}-r_{f}}{\sigma_{p}} \\
\text { Subject to : } & \sigma_{p}^{2}=\frac{1}{c}\left(1+\frac{\left(c \mu_{p}-b\right)^{2}}{a c-b^{2}}\right), \\
& l^{T} w=1, \\
& 0 \leq w_{i} \leq 1 \forall i=1,2, \ldots, n .
\end{array}
$$

Sharpe ratio efficient opportunity $(S R E O)$ portfolio is a portfolio with optimal weight vector of $S R E O M$. 


\subsection{Equally weighted portfolio}

Investors select equally weighted portfolio to keep diversification. However, equally weight portfolio may or may not be optimal portfolio. Let $P_{e w}$ be equally weighted portfolio. Then expected return and risk of $P_{e w}$ are given by:

$\mu_{e w}=\frac{l^{T} \mu}{n}$ and $\sigma_{e w}=\frac{\sqrt{l^{T} \Sigma l}}{n}$. Thus, Sharpe ratio of equally weighted portfolio is defined by:

$S R_{e w}=\frac{l^{T} \mu-r_{f}}{\sqrt{l^{T} \Sigma l}}$.

\subsection{Order three complete portfolio}

The return of order three complete portfolio $P_{3 c}$ is defined by $r_{3 c}=\frac{w_{p}+\delta}{3} r_{f}+\frac{\rho+1-\delta}{3} r_{m}+\frac{2-\rho-w_{p}}{3} r_{p}$. Without loss of generality, let us assume that $w_{p}$ is given. Then one can find $\delta$ using weight-variance regression model and $\rho$ using order three weight equation. Therefore, for a given $w_{p}$ there exist $\delta$ and $\rho$ which satisfy order three weight equation.

\section{Weight-variance regression model}

The regression model $\ln \left(\sigma_{p}\right)=\alpha+\beta \sigma_{p m}^{2}+\epsilon_{p}$ is called weight-variance regression model. We use feasible generalized least square $F G L S$ regression method to estimate the parameters $\alpha$ and $\beta$ if variance of residual square is not constant. Solve for optimal solution $\frac{1-\delta}{1-w_{p}}$ of $\min \left(\ln \left(\sigma_{m}\right)+\ln \left(\frac{1-\delta}{1-w_{p}}\right)-\hat{\alpha}-\hat{\beta} \sigma_{m}^{4}\left(\frac{1-\delta}{1-w_{p}}\right)^{2}\right)^{2}$. Therefore, $\frac{1-\delta}{1-w_{p}}$ can be estimated by the help of weight-variance regression model. Note that $\frac{1-\delta}{1-w_{p}}=\frac{1}{\sigma_{m}^{2} \sqrt{2 \hat{\beta}}}$. Suppose that there is no opportunity cost between the two fundamental portfolios. That is, fundamental portfolios have the same level of risk and expected return. This assumption is correct because in finance there is no free lunch or arbitrage opportunity. Define expected return of risky fundamental portfolio by $\mu_{c}=$ $w_{p} \mu_{p}+\left(1-w_{p}\right) \mu_{m}$. Similarly, define risk of risky fundamental portfolio by $\sigma_{c}^{2}=w_{p}^{2} \sigma_{p}^{2}+\left(1-w_{p}\right)^{2} \sigma_{m}^{2}+2 w_{p}\left(1-w_{p}\right) \sigma_{p m}$. Notation: $\mu_{p}=\mu^{T} w$ and $\sigma_{p}^{2}=w^{T} \Sigma w$.

Now one can build a model based on efficiency condition, Sharpe ratio error condition and weight-variance regression model. Define $\sigma_{p m}=\left(\sigma_{1 m}, \sigma_{2 m}, \ldots, \sigma_{n m}\right)^{T}$. Therefore, we have the following Sharpe variance model $(S V M)$.

$$
S V M: \min _{w, w_{p}} \sigma_{c}^{2}
$$

Subject to : $\sigma_{c}=\sqrt{z}\left(\mu_{c}-r_{f}\right)$,

$$
\begin{aligned}
& \ln \left(\sigma_{p}\right)=\hat{\alpha}+\hat{\beta} \sigma_{p m}^{2}, \\
& l^{T} w=1, \\
& 0 \leq w_{p}, w_{i} \leq 1 \forall i=1,2, \ldots, n .
\end{aligned}
$$

$S V$ is called Sharpe variance portfolio.

\section{Treynor-Sharpe regression model}

Define Treynor ratio $t r_{i}$ of security $i$ by $t r_{i}=$ $\frac{\mu_{i}-r_{f}-\beta_{i}\left(\mu_{m}-r_{f}\right)}{\beta_{i}}$. Similarly, define Sharpe ratio $s r_{i}$ of security $i$ by $s r_{i}=\frac{\mu_{i}-r_{f}}{\sigma_{i}}$. Then the model

$$
t r_{i}=a+b s r_{i}+\epsilon_{i}
$$

is called Treynor-Sharpe regression model. The line $t r_{i}=\hat{a}+$ $\hat{b} s r_{i}$ called Treynor-Sharpe regression line. We have seen the case $\rho=w_{p}$ for equivalent portfolios of order two. Let us consider the opposite case of $\rho=w_{p}$. Suppose that $w_{p} r_{f}+$ $\left(1-w_{p}\right) r_{m}=\rho r_{p}+(1-\rho) r_{m}$ and $\rho \neq w_{p}$.

Let us consider three investment portfolio construction scenarios. Let $P_{f r}, P_{m r}$ and $P_{f m r}$ denote free-risky portfolio, market-risky portfolio and free-market-risky portfolio, respectively. Consider the following return condition of these portfolios. Suppose that $n=w_{p} r_{f}+\left(1-w_{p}\right) r_{p}=\rho r_{m}+(1-\rho) r_{p}=$ $\delta r_{f}+(1-\delta) r_{m}$. The condition $w_{p} r_{f}+\left(1-w_{p}\right) r_{p}=$ $\rho r_{m}+(1-\rho) r_{p}=\delta r_{f}+(1-\delta) r_{m}$ is called order three opportunity condition.

Lemma 5.1. If order three opportunity condition is valid, then $\rho=\frac{(1-\delta) w_{p}}{w_{p}-\delta}$. This equation is called order three weight equation.

Proof. Suppose that order three opportunity condition is valid. Then $r_{p}=\frac{n-w_{p} r_{f}}{1-w_{p}}$. This implies that $n=\rho r_{m}+(1-$ $\rho) r_{p}=\rho r_{m}+(1-\rho) \frac{n-w_{p} r_{f}}{1-w_{p}}$. Solve for $n$. Thus, $\frac{\rho\left(1-w_{p}\right) r_{m}+(\rho-1) w_{p} r_{f}}{\rho-w_{p}}=\delta r_{f}+(1-\delta) r_{m}$. Solve for $\rho$ and express it in terms of $w_{p}$ and $\delta$. Hence the proof followed.

Lemma 5.2. If order three opportunity condition is valid, then $\left(1-w_{p}\right) \sigma_{p}^{2}-(1-\delta) \sigma_{p m}=0$. This equation is called weightvariance equation.

Proof. Suppose that order three opportunity condition is valid. Then $\rho^{2} \sigma_{m}^{2}+(1-\rho)^{2} \sigma_{p}^{2}+2(1-\rho) \rho \sigma_{p m}=(1-\delta)^{2} \sigma_{m}^{2}$ and $\sigma_{p}^{2}=\left(\frac{1-\delta}{1-w_{p}}\right)^{2} \sigma_{m}^{2}$. Use $\rho=\frac{(1-\delta) w_{p}}{w_{p}-\delta}$ to solve for $\sigma_{p m}$. Thus, $\sigma_{p m}=\frac{1-\delta}{1-w_{p}} \sigma_{m}^{2}$. Hence the proof followed.

Lemma 5.3. If order three opportunity condition holds, then $\left(w_{p}-\rho\right) \frac{1-\delta}{1-w_{p}}=-\rho$.

Proof. Suppose that order three opportunity condition holds. Then $\left(w_{p}-\rho\right) \frac{1-\delta}{1-w_{p}}=\left(w_{p}-\frac{(1-\delta) w_{p}}{w_{p}-\delta}\right) \frac{1-\delta}{1-w_{p}}$. This implies that $\left(w_{p}-\rho\right) \frac{1-\delta}{1-w_{p}}=-\frac{(1-\delta) w_{p}}{w_{p}-\delta}=-\rho$. Hence the proof followed.

Lemma 5.4. If order three opportunity condition holds, then both risky portfolio $p$ and market portfolio $m$ have equal Sharpe ratio.

Proof. Suppose that order three opportunity condition holds. Then $\mu_{p}=\frac{w_{p} r_{f}-\rho \mu_{m}}{w_{p}-\rho}$. This implies that $\mu_{p}-r_{f}=$ $\frac{w_{p} r_{f}-\rho \mu_{m}}{w_{p}-\rho}-r_{f}=\rho \frac{r_{f}-\mu_{m}}{w_{p}-\rho}$. Clearly, $\sigma_{p}=\frac{(1-\delta)}{1-w_{p}} \sigma_{m}$. This implies that $\frac{\mu_{p}-r_{f}}{\sigma_{p}}=\rho \frac{r_{f}-\mu_{m}}{\left(w_{p}-\rho\right) \frac{(1-\delta)}{1-w_{p}} \sigma_{m}}$. Thus, $\frac{\mu_{p}-r_{f}}{\sigma_{p}}=\frac{\mu_{m}-r_{f}}{\sigma_{m}}$. Hence the proof followed. 
Lemma 5.5. If order three opportunity condition holds, then both risky portfolio $p$ and market portfolio $m$ have equal Treynor ratio.

Proof. Suppose that order three opportunity condition holds. Let $t_{p}$ and $t_{m}$ be Treynor ratio of portfolio $p$ and market portfolio $m$, respectively. Clearly, $t_{m}=0$. Claim: To show that $t_{p}=0 . \quad t_{p}=\sigma_{m}^{2}\left(\frac{\mu_{p}-r_{f}}{\sigma_{p m}}\right)+\left(r_{f}-\mu_{m}\right)$. Clearly, $\sigma_{p m}=$ $\frac{(1-\delta)}{1-w_{p}} \sigma_{m}^{2}$. This implies that $t_{p}=\frac{1-w_{p}}{1-\delta}\left(\mu_{p}-r_{f}\right)+\left(r_{f}-\mu_{m}\right)$. Since $\mu_{p}-r_{f}=\rho \frac{r_{f}-\mu_{m}}{w_{p}-\rho}, t_{p}=\left(-r_{f}+\mu_{m}\right)+\left(r_{f}-\mu_{m}\right)=0$. Hence the proof followed.

Theorem 5.6. If order three opportunity condition holds, then both order three complete portfolio $P_{3 c}$ and market portfolio $m$ have equal Treynor ratio.

Proof. Suppose that order three opportunity condition holds. Then $r_{3 c}=\rho r_{m}+(1-\rho) r_{p}$. Define Treynor ratio $t_{3 c}$ of order three opportunity complete portfolio by:

$$
t_{3 c}=\frac{\alpha_{3 c}}{\beta_{3 c}}=\frac{\mu_{3 c}-r_{f}-\beta_{3 c}\left(\mu_{m}-r_{f}\right)}{\beta_{3 c}} .
$$

Similarly, Treynor ratio $t_{p}$ of risky portfolio is defined by:

$$
t_{p}=\frac{\alpha_{p}}{\beta_{p}}=\frac{\mu_{p}-r_{f}-\beta_{p}\left(\mu_{m}-r_{f}\right)}{\beta_{p}} .
$$

It follows from equations (84) and (85) that

$$
t_{p}=\frac{\mu_{p}-r_{f}}{\beta_{p}}+t_{3 c}-\left(\frac{(1-\rho) \mu_{p}+\rho \mu_{m}-r_{f}}{(1-\rho) \beta_{p}+\rho}\right)
$$

Consider the following result.

$$
\begin{aligned}
& \frac{\mu_{p}-r_{f}}{\beta_{p}}-\left(\frac{(1-\rho) \mu_{p}+\rho \mu_{m}-r_{f}}{(1-\rho) \beta_{p}+\rho}\right) \\
& =\frac{\left(\mu_{p}-r_{f}\right)\left((1-\rho) \beta_{p}+\rho\right)-\beta_{p}\left((1-\rho) \mu_{p}+\rho \mu_{m}-r_{f}\right)}{\beta_{p}\left((1-\rho) \beta_{p}+\rho\right)} \\
& =\rho\left(\frac{\mu_{p}-r_{f}-\beta_{p}\left(\mu_{m}-r_{f}\right)}{\beta_{p}\left((1-\rho) \beta_{p}+\rho\right)}\right) \\
& =\left(\frac{\rho}{(1-\rho) \beta_{p}+\rho}\right) t_{p} .
\end{aligned}
$$

This implies that

$\frac{\mu_{p}-r_{f}}{\beta_{p}}-\left(\frac{(1-\rho) \mu_{p}+\rho \mu_{m}-r_{f}}{(1-\rho) \beta_{p}+\rho}\right)=\left(\frac{\rho}{(1-\rho) \beta_{p}+\rho}\right) t_{p}$.

Substitute the equation (87) in to equation (86) to get the equation

$$
t_{p}=\left(\frac{\rho}{(1-\rho) \beta_{p}+\rho}\right) t_{p}+t_{3 c}
$$

Clearly,

$$
t_{3 c}=\left(\frac{1-\rho}{(1-\rho) \beta_{p}+\rho}\right) t_{p} .
$$

Since $t_{p}=0$, we get $t_{3 c}=0$. Hence the proof followed.

Theorem 5.7. Let $s_{3 c}$ be Sharpe ratio of order three complete portfolio $P_{3 c}$. Suppose that $0<w_{p}<1$. If order three opportunity condition holds, then $s_{3 c}=s_{p}$.
Suppose that order three opportunity condition holds. Then $\sigma_{p}^{2}=\left(\frac{1-\delta}{1-w_{p}}\right)^{2} \sigma_{m}^{2}$ and $\sigma_{p m}=\frac{1-\delta}{1-w_{p}} \sigma_{m}^{2}$. Consider the ratio of complete and risky portfolios Sharpe ratios. Suppose that $\sigma_{3 c}^{2} \neq 0$. Define $g_{\rho}$ and $s_{\rho}$ by: $g_{\rho}=\left(\frac{(1-\rho) \mu_{p}+\rho \mu_{m}-r_{f}}{\mu_{p}-r_{f}}\right)$ and $s_{\rho}=\sqrt{\frac{\sigma_{p}^{2}}{(1-\rho)^{2} \sigma_{p}^{2}+\rho^{2} \sigma_{m}^{2}+2 \rho(1-\rho) \sigma_{p m}}}=\frac{1}{1-w_{p}}$. Clearly, $\frac{s_{3 c}}{s_{p}}=g_{\rho} s_{\rho}$ and $(1-\rho) \mu_{p}+\rho \mu_{m}-r_{f}=(1-\delta)\left(\mu_{m}-r_{f}\right)$. Note that $\mu_{p}-r_{f}=-\rho \frac{\mu_{m}-r_{f}}{w_{p}-\rho}$. It is obvious that $s_{3 c}=s_{p}$. Hence the proof followed.

Theorem 5.8. Suppose that $\rho \neq w_{p}$. $w_{p} r_{f}+\left(1-w_{p}\right) r_{m}=$ $\rho r_{p}+(1-\rho) r_{m}$ if and only if $r_{p}=\frac{w_{p}}{\rho} r_{f}+\left(1-\frac{w_{p}}{\rho}\right) r_{m}$.

Proof. Suppose that $w_{p} r_{f}+\left(1-w_{p}\right) r_{m}=\rho r_{p}+(1-\rho) r_{m}$ and $\rho \neq w_{p}$. Set $r_{h}=w_{p} r_{f}+\left(1-w_{p}\right) r_{m}$. First solve for $r_{m}$ in terms of $r_{h}$. Thus, $r_{m}=\frac{r_{h}-w_{p} r_{f}}{1-w_{p}}=\frac{r_{h}-\rho r_{p}}{1-\rho}$. Solve for $r_{h}$ from $\frac{r_{h}-w_{p} r_{f}}{1-w_{p}}=\frac{r_{h}-\rho r_{p}}{1-\rho}$. Clearly, $r_{h}=\frac{w_{p}(1-\rho) r_{f}+\left(w_{p}-1\right) \rho r_{p}}{w_{p}-\rho}$. This implies that $w_{p} r_{f}+\left(1-w_{p}\right) r_{m}=\frac{w_{p}(1-\rho) r_{f}+\left(w_{p}-1\right) \rho r_{p}}{w_{p}-\rho}$. Now solve for $r_{p}$. Hence the result follows immediately. Conversely, suppose that $r_{p}=\frac{w_{p}}{\rho} r_{f}+\left(1-\frac{w_{p}}{\rho}\right) r_{m}$. Then $\rho r_{p}+(1-\rho) r_{m}=\rho\left(\frac{w_{p}}{\rho} r_{f}+\left(1-\frac{w_{p}}{\rho}\right) r_{m}\right)+(1-\rho) r_{m}$. The result follows immediately. Hence the proof followed.

Theorem 5.9 (Fundamental theorem of order two zero investment opportunity). Suppose that a point $\left(s r_{p}, t r_{p}\right)$ lies on Treynor-Sharpe regression line and $\mu_{m} \neq r_{f}$. If $w_{p} r_{f}+(1-$ $\left.w_{p}\right) r_{m}=\rho r_{p}+(1-\rho) r_{m}$, then $w_{p}=\rho$.

Proof. Suppose that the a point $\left(s r_{p}, t r_{p}\right)$ lies on TreynorSharpe regression line and $\mu_{m} \neq r_{f}$. Then $t r_{p}=\hat{a}+\hat{b} s r_{p}$. Apply proof by contradiction method to prove this theorem. Suppose $w_{p} \neq \rho$. Then $w_{p}>\rho$ or $w_{p}<\rho$. Case one: Suppose $w_{p}>\rho$. It follows from theorem 5.8 that $r_{p}=$ $\frac{w_{p}}{\rho} r_{f}+\left(1-\frac{w_{p}}{\rho}\right) r_{m}$. This implies that $\beta_{p}=\left(1-\frac{w_{p}}{\rho}\right)$, $\mu_{p}=\frac{w_{p}}{\rho} r_{f}+\left(1-\frac{w_{p}}{\rho}\right) \mu_{m}$ and $\sigma_{p}=\left(\frac{w_{p}}{\rho}-1\right) \sigma_{m}$. Clearly, $t r_{p}=0$. Thus, $\mu_{m}=r_{f}$. This contradicts with the given fact $\mu_{m} \neq r_{f}$. Therefore, $w_{p}>\rho$ is false. Case two: Suppose $w_{p}<\rho$. Similarly, it follows from theorem 5.8 that $r_{p}=\frac{w_{p}}{\rho} r_{f}+\left(1-\frac{w_{p}}{\rho}\right) r_{m}$. This implies that $\beta_{p}=\left(1-\frac{w_{p}}{\rho}\right)$, $\mu_{p}=\frac{w_{p}}{\rho} r_{f}+\left(1-\frac{w_{p}}{\rho}\right) \mu_{m}$ and $\sigma_{p}=\left(1-\frac{w_{p}}{\rho}\right) \sigma_{m}$. Again $t r_{p}=0$. Thus, $\mu_{m}=r_{f}$. This contradicts with the given fact $\mu_{m} \neq r_{f}$. Therefore, $w_{p}<\rho$ is false. This shows that $w_{p}=\rho$. Hence the proof followed.

\section{General order three opportunity model}

Use weight-variance regression model to estimate $\frac{1-\delta}{1-w_{p}}$. Thus, $(1-\delta) \sigma_{m}^{2} \sqrt{2 \frac{\operatorname{cov}\left(\ln \left(\sigma_{p}\right), \sigma_{p m}^{2}\right)}{\operatorname{var}\left(\sigma_{p m}^{2}\right)}}=\left(1-w_{p}\right)$. Define $\hat{u}=\sigma_{m}^{2} \sqrt{2 \frac{\operatorname{cov}\left(\ln \left(\sigma_{p}\right), \sigma_{p m}^{2}\right)}{\operatorname{var}\left(\sigma_{p m}^{2}\right)}}$. Clearly, $(1-\delta) \hat{u}=\left(1-w_{p}\right)$.

Theorem 6.1. The following equations are necessary conditions for order three opportunity condition.

1. $\beta_{p}=\frac{1-\delta}{1-w_{p}}$, 
2. $\mu_{p}-r_{f}=\beta_{p}\left(\mu_{m}-r_{f}\right)$,

3. $\ln \left(\sigma_{p}\right) \approx \hat{\alpha}+\hat{\beta} \sigma_{p m}^{2}$.

The following model is called general order three opportunity model $(G O O M)$.

$$
\text { GOOM : } \min _{w} \sigma_{p}^{2}
$$

Subject to :

$$
\begin{aligned}
& \beta_{p}=\frac{1}{\sigma_{m}^{2} \sqrt{2 \hat{\beta}}}, \\
& \mu_{p}-r_{f}=\beta_{p}\left(\mu_{m}-r_{f}\right), \\
& \ln \left(\sigma_{p}\right)=\hat{\alpha}+\hat{\beta} \sigma_{p m}^{2}, \\
& l^{T} w=1, \\
& 0 \leq w_{i} \leq 1 \forall i=1,2, \ldots, n .
\end{aligned}
$$

Order three $(O T H)$ portfolio is a portfolio with optimal weight vector of $G O O M$.

\section{Order three asset allocation model}

$$
\text { Define } d_{b}=\frac{1}{c}\left(1+\frac{\left(c\left(\frac{\delta\left(\mu_{m}-r_{f}\right)-\mu_{m}+w_{p} r_{f}}{w_{p}-1}\right)-b\right)^{2}}{a c-b^{2}}\right) \text {. }
$$

Definition 7.1 (Order three efficiency condition). The equation

$$
\left(\frac{1-\delta}{1-w_{p}}\right)^{2} \sigma_{m}^{2}=d_{b}
$$

is called order three efficiency condition.

$$
\text { Define } d_{k}=\left(w_{p}^{2}\left(\frac{1-\delta}{1-w_{p}}\right)^{2}+\left(1-w_{p}\right)^{2}+2 w_{p}(1-\delta)\right) \text {. }
$$

Definition 7.2 (Order three Sharpe ratio error condition). The equation

$$
d_{k}=\left(w_{p}^{2}\left(\frac{1-\delta}{1-w_{p}}\right)^{2}+\left(1-w_{p}\right)^{2}+2 w_{p}(1-\delta)\right)
$$

is called order three Sharpe ratio error condition.

Theorem 7.3. If efficiency condition, Sharpe ratio error condition and order three zero opportunity condition hold, then order three efficiency condition and order three Sharpe ratio error condition hold.

Proof. Suppose that efficiency condition, Sharpe ratio error condition and order three zero opportunity condition hold. Then

$$
w^{T} \Sigma w=\frac{1}{c}\left(1+\frac{\left(c \mu^{T} w-b\right)^{2}}{a c-b^{2}}\right)
$$

and

$$
\sigma_{S P_{2}}=\frac{\overline{r_{S P_{2}}}-r_{f}}{\mu_{m}-r_{f}} \sigma_{m} .
$$

Clearly, the following equation follows from order three zero opportunity condition.

$$
\delta=\left(\frac{\mu_{p}-r_{f}}{\mu_{m}-r_{f}}\right) w_{p}-\left(\frac{\mu_{p}-\mu_{m}}{\mu_{m}-r_{f}}\right) .
$$

Solve for $\mu_{p}$ from equation (95) to find equation (96).

$$
\mu_{p}=\frac{\delta\left(\mu_{m}-r_{f}\right)-\mu_{m}+w_{p} r_{f}}{w_{p}-1} .
$$

Clearly,

$$
\sigma_{S P_{2}}^{2}=w_{p}^{2} \sigma_{p}^{2}+\left(1-w_{p}\right)^{2} \sigma_{m}^{2}+2 w_{p}\left(1-w_{p}\right) \sigma_{p m} .
$$

It follows from order three zero opportunity condition that

$$
\sigma_{p}^{2}=\left(\frac{1-\delta}{1-w_{p}}\right)^{2} \sigma_{m}^{2}
$$

and

$$
\sigma_{p m}=\frac{1-\delta}{1-w_{p}} \sigma_{m}^{2} .
$$

Substitute equations (98) and (99) in to the equation (97) to get equation (100).

$$
\sigma_{S P_{2}}^{2}=\sigma_{m}^{2}\left(w_{p}^{2}\left(\frac{1-\delta}{1-w_{p}}\right)^{2}+\left(1-w_{p}\right)^{2}+2 w_{p}(1-\delta)\right) .
$$

Consider the following equation.

$$
\begin{aligned}
\overline{r_{S P_{2}}}-r_{f} & =w_{p} \mu_{p}+\left(1-w_{p}\right) \mu_{m}-r_{f} \\
& =w_{p}\left(\frac{\delta\left(\mu_{m}-r_{f}\right)-\mu_{m}+w_{p} r_{f}}{w_{p}-1}\right)+\left(1-w_{p}\right) \mu_{m} \\
& -r_{f} \\
& =\left(\frac{(1+\delta) w_{p}-1-w_{p}^{2}}{w_{p}-1}\right)\left(\mu_{m}-r_{f}\right)
\end{aligned}
$$

Substitute equation (101) in to the equation (94) to get equation (102).

$$
\sigma_{S P_{2}}^{2}=\sigma_{m}^{2}\left(\frac{(1+\delta) w_{p}-1-w_{p}^{2}}{w_{p}-1}\right)^{2} .
$$

It follows from equations (100) and (102) that

$$
\left(\frac{(1+\delta) w_{p}-1-w_{p}^{2}}{w_{p}-1}\right)^{2}=d_{k}
$$

Substitute equations (96) and (98) in to the equation (93) to get equation (104)

$$
\left(\frac{1-\delta}{1-w_{p}}\right)^{2} \sigma_{m}^{2}=\frac{1}{c}\left(1+\frac{\left(c\left(\frac{\delta\left(\mu_{m}-r_{f}\right)-\mu_{m}+w_{p} r_{f}}{w_{p}-1}\right)-b\right)^{2}}{a c-b^{2}}\right)
$$

Hence the proof followed.

Order three asset allocation model $(O A A M)$ is given by:

$$
\begin{aligned}
O A A M: \min _{w_{p}, \delta, \rho} & \left(\rho\left(w_{p}-\delta\right)+(\delta-1) w_{p}\right)^{2} \\
\text { Subject to : } & \left(\frac{1-\delta}{1-w_{p}}\right)^{2} \sigma_{m}^{2}=d_{b}, \\
& \left(\frac{(1+\delta) w_{p}-1-w_{p}^{2}}{w_{p}-1}\right)^{2}=d_{k}, \\
& 0 \leq w_{p}, \delta, \rho \leq 1 .
\end{aligned}
$$




\subsection{Sharpe ratio process}

Sharpe ratio process (vector) is defined by $s \vec{r}_{t p}=$ $\left(\frac{\mu_{2 p}-r_{f}}{\sigma_{2 p}}, \frac{\mu_{3 p}-r_{f}}{\sigma_{3 p}}, \ldots, \frac{\mu_{t p}-r_{f}}{\sigma_{t p}}\right)^{\tau}$, where $\tau$ is an investment horizon.

\section{Results}

Secondary data on stocks return is used for demonstration purpose. In this research, we use 14 oct 2019- 7 oct 2020 daily return historical data of USA 22 stock companies from yahoo finance. We also use one year bond rate $r_{f}=0.11 \%$ (daily bond rate $r_{f}=0.000003$ ) for empirical study. All empirical results of this study is for long selling option. In this study, we have not included short selling empirical results. We have used global search in MatLab to solve optimization problems of the study. Regression analysis is done by the help of $R$. In this research, we have constructed Sharpe variance portfolio $(S V)$, order three portfolio $(O T H)$ and complete portfolio $(C P)$. We solve Sharpe variance model $(S V M)$ to find optimal weights of Sharpe variance portfolio $(S V)$. We use general order three opportunity model $(G O O M)$ to solve for optimal weights of order three portfolio $(O T H)$. Return of complete portfolio is given by $r_{t c p}=w_{f}^{*} r_{f}+w_{p}^{*} r_{t O T H}+w_{m}^{*} r_{t m}$, where $r_{t O T H}$ is rate of return of order three portfolio $(O T H)$ at time $t, w_{f}^{*}=$ $\frac{w_{p}+\delta}{3}, w_{p}^{*}=\frac{2-\rho-w_{p}}{3}$ and $w_{m}^{*}=\frac{\rho+1-\delta}{3}$. We apply order three asset allocation model $(O A A M)$ to solve for $w_{p}, \delta$ and $\rho$. The empirical study of this research gives $w_{p}=0, \delta=0.3661$ and $\rho=0$. Thus, we have $w_{f}^{*}=0.122, w_{p}^{*}=0.6667$ and $w_{m}^{*}=$ 0.2113 . Let us see weights of Sharpe variance portfolio $(S V)$, order three portfolio $(O T H)$ and complete portfolio $(C P)$ as given in Table 2.

Table 2. Summary of optimal weight of SV, OTH and CP portfolios

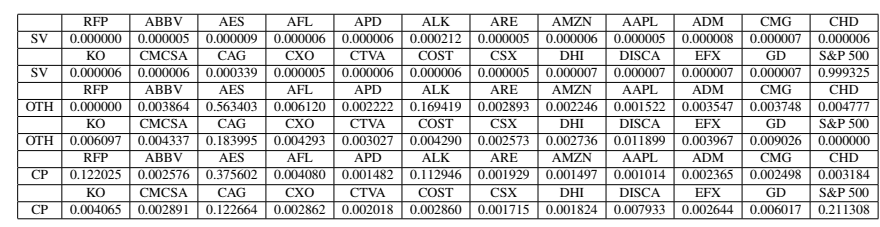

Observe from Table 2 that $S V$ is very close to market portfolio $S \& P 500$. Efficiency condition is not considered for $S V$ construction. Note that order three opportunity condition is considered for $O T H$ construction. We assume that there is no opportunity cost between two investment options. Thus, OTH is more realistic model than $S V$. Since $O T H$ is not complete portfolio, we want to construct complete portfolio based on order three zero opportunity condition and efficiency condition. Therefore, $C P$ is realistic portfolio for investors who consider order three zero opportunity condition and efficiency condition.
Table 3. Summary of performance metrics of portfolios.

\begin{tabular}{|c|c|c|c|c|c|c|c|}
\hline Company & $\sigma$ & $\mu$ & $\frac{\mu-r_{f}}{\sigma}$ & $\beta$ & $\alpha$ & Sharpe error & $\frac{\alpha}{\beta}$ \\
\hline ABBV & 0.021013 & 0.000891 & 0.042269 & 0.715497 & 0.000314 & 0.000008 & 0.0004 \\
\hline AES & 0.036228 & 0.001311 & 0.036094 & 1.169336 & 0.000370 & 0.000002 & 0.0003 \\
\hline AFL & 0.035262 & -0.000698 & -0.019880 & 1.215145 & -0.001676 & 0.002918 & -0.0014 \\
\hline APD & 0.026578 & 0.001746 & 0.065588 & 1.038224 & 0.000910 & 0.000402 & 0.0009 \\
\hline ALK & 0.047673 & -0.001187 & -0.024957 & 1.435507 & -0.002341 & 0.006320 & -0.0016 \\
\hline ARE & 0.026188 & 0.000582 & 0.022103 & 1.008092 & -0.000230 & 0.000115 & -0.0002 \\
\hline AMZN & 0.022913 & 0.002723 & 0.118716 & 0.666875 & 0.002185 & 0.002485 & 0.0033 \\
\hline AAPL & 0.028240 & 0.003098 & 0.109586 & 1.113393 & 0.002202 & 0.002975 & 0.0020 \\
\hline ADM & 0.024454 & 0.001039 & 0.042384 & 0.954839 & 0.000271 & 0.000011 & 0.0003 \\
\hline CMG & 0.030650 & 0.002217 & 0.072230 & 0.847992 & 0.001534 & 0.000816 & 0.0018 \\
\hline CHD & 0.020758 & 0.001061 & 0.050967 & 0.536940 & 0.000627 & 0.000057 & 0.0012 \\
\hline KO & 0.021209 & -0.000067 & -0.003304 & 0.787616 & -0.000702 & 0.000533 & -0.0009 \\
\hline CMCSA & 0.023446 & 0.000249 & 0.010471 & 0.877408 & -0.000458 & 0.000285 & -0.0005 \\
\hline CAG & 0.023943 & 0.001479 & 0.061628 & 0.414378 & 0.001143 & 0.000241 & 0.0028 \\
\hline CXO & 0.040978 & -0.000734 & -0.017983 & 1.280099 & -0.001764 & 0.003683 & -0.0014 \\
\hline CTVA & 0.032291 & 0.001123 & 0.034674 & 1.054730 & 0.000274 & 0.000005 & 0.0003 \\
\hline COST & 0.017816 & 0.000959 & 0.053636 & 0.599532 & 0.000475 & 0.000060 & 0.0008 \\
\hline CSX & 0.028728 & 0.000969 & 0.033617 & 1.166044 & 0.000030 & 0.000008 & 0.0000 \\
\hline DHI & 0.035733 & 0.002144 & 0.059912 & 1.167007 & 0.001205 & 0.000464 & 0.0010 \\
\hline DISCA & 0.031693 & -0.000399 & -0.012686 & 0.889315 & -0.001115 & 0.001802 & -0.0013 \\
\hline EFX & 0.025100 & 0.000785 & 0.031146 & 0.869206 & 0.000084 & 0.000018 & 0.0001 \\
\hline GD & 0.024822 & -0.000546 & -0.022129 & 0.920442 & -0.001288 & 0.001561 & -0.0014 \\
\hline S\&P 500 & 0.021460 & 0.000805 & 0.037381 & 1.000000 & 0.000000 & 0.000000 & 0.0000 \\
\hline
\end{tabular}

Consider table 3 to observe the following results. The minimum standard deviation corresponds to COST. AAPL has maximum expected return. The company with maximum Sharpe ratio is $A M Z N$. $C A G$ has smallest beta where as $A A P L$ has maximum alpha. Clearly, the market index has zero Sharpe error. Finally, $A M Z N$ has maximum alpha to beta ratio(Treynor ratio). Opportunity models require estimation of weight-variance model parameters. We assume that portfolio follows weight-variance regression line. That is, natural logarithm of standard deviation of portfolio is assumed as linear function of square of co-variance between portfolio return and market index return. Opportunity models take weightvariance regression line as a constraint. Before applying ordinary least $(O L S)$ square parameters estimation method, one should check the validity of $(O L S)$ assumption. If all assumptions of $(O L S)$ hold, then one can apply $(O L S)$ to estimate parameters of linear regression. We want to test weight-variance model $(O L S)$ assumptions validity using $\mathrm{R}$ program. Ordinary least square method has some basic assumption. Some of these assumptions are linear relation ship between explanatory and response variable, constant variance of residual square, error is normally distributed with expected value zero and constant variance, explanatory variables should be independent to each other. Let us consider hetroscedasticity problem testing methods. In this research, we apply white test and alternative white test to check the hetroscedasticity problem. Once weight-variance regression line parameters are estimated, use the estimated parameters in opportunity models to select portfolio based on zero opportunity cost.

Consider the following scatter plot. 


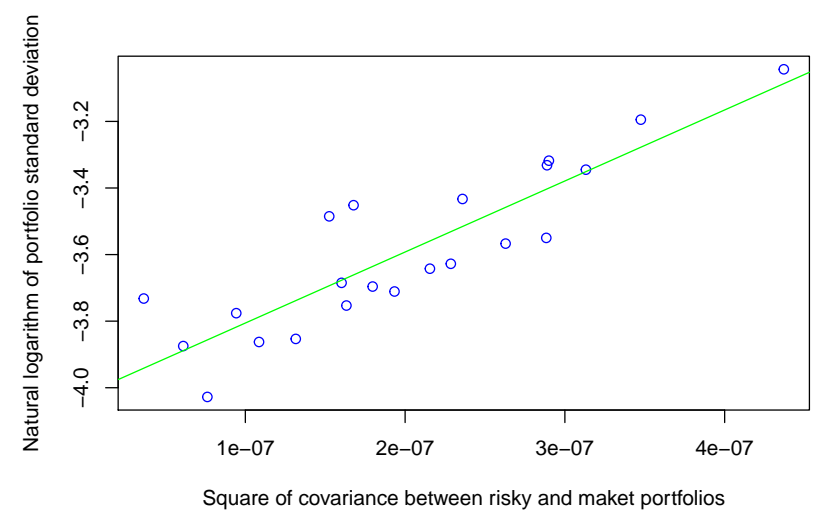

Figure 1. Scatter plot of logarithm of standard deviation against square of co-variance

As shown in figure 1, there is linear relation ship between natural logarithm of risky portfolio standard deviation and square of co-variance between risky and market portfolio. Both white and alternative white test hetroscedasticity problem testing methods show that there is no statistically significant hetroscedasticity problem. Therefore, we can apply $O L S$ to estimate weight-variance model parameters. The required weightvariance model equation is given by:

$$
\ln \left(\sigma_{p}\right)=-4.031+2.1206 e+06 \sigma_{p m}^{2} .
$$

The next step is estimating the value of $\frac{1-\delta}{1-w}=\frac{1}{\sigma_{m}^{2} \sqrt{2 \hat{\beta}}}$. Use $\hat{\beta}=2.121\left(10^{6}\right)$ and $\sigma_{m}=0.02145974$ to find $\frac{1-\delta}{1-w}$. Hence $\frac{1-\delta}{1-w}=1.054398$. Let us consider basic portfolios and their optimal weight vector. In practice, investors use long selling assuming that short selling is not allowed. Thus, we assume weights of securities as non-negative. Treynor ratio maximization model $(T R M M)$ is given by:

$$
\begin{array}{cl}
\text { TRMM } & \max \frac{\alpha_{p}}{\beta_{p}} \\
\text { subject to }: & l^{T} w=1 .
\end{array}
$$

Now we want to test the assumption $\rho=w_{P}$ for second order zero opportunity portfolio. It is proved that $t r_{p}=\hat{a}+$ $\hat{b} s r_{p}$ is sufficient condition for $\rho=w_{p}$. Thus, we should test the linear relation between Treynor ratio and Sharpe ratio using linear regression. One can observe from figure 2 that there is positive linear relation ship between Treynor ratio and Sharpe ratio.
Treynor-Sharpe regression line

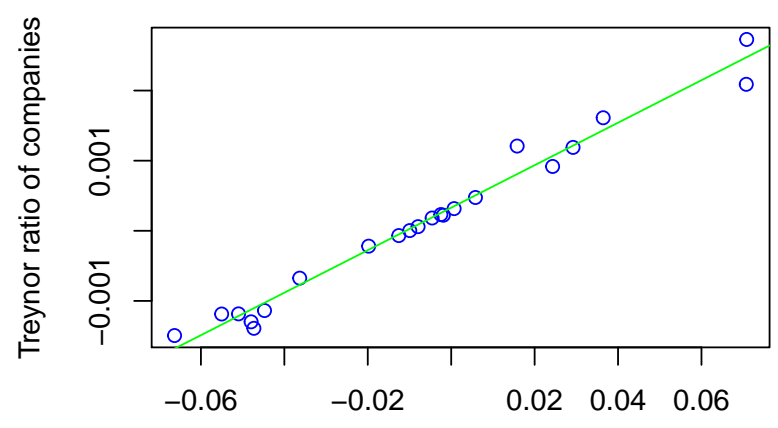

Sharpe ratio of companies

Figure 2. Plot of Treynor ratio against Sharpe ratio

Figure 2 shows that there is positive linear relation ship between Sharpe ratio of companies and Treynor ratio of companies. Thus, we assume portfolio follows Treynor-Sharpe regression line. As a result, we assume $\rho=w_{P}$.

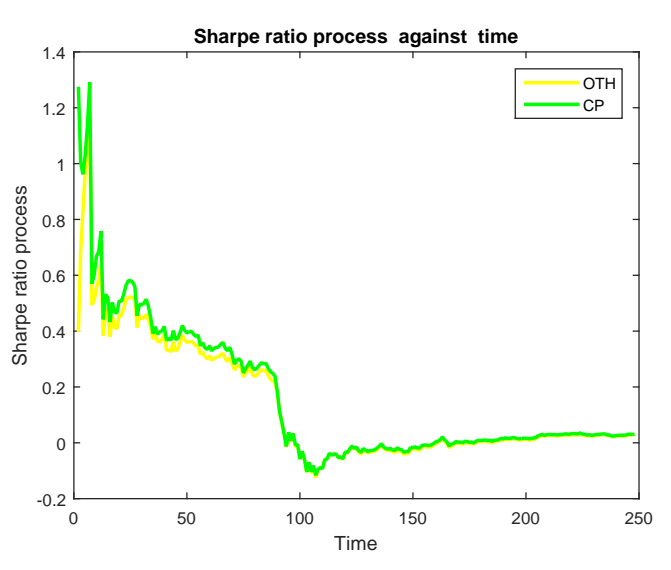


Let consider the performance of $O T H$ and $C P$. Note that $C P$ is more diversified portfolio than $O T H$. We observe from Figure 3 that $C P$ outperforms $O T H$ based on Sharpe ratio process.

\section{Discussion}

Mean variance model is applicable only either expected return of portfolio or variance of portfolio return is given. But in reality both expected return and variance of portfolio are not known. Therefore, mean variance model has no supportive evidence unless either expected return of portfolio or variance of portfolio return is given. Another problem of mean variance model is it does not consider some logical constraints like opportunity cost and arbitrage opportunity constraints. By market efficiency hypothesis, information about securities prices is available in public. Thus, there is no arbitrage opportunity and opportunity cost. In this research, we have investigated two portfolio selection and asset allocation strategies based on the evidence that there is positive linear relation ship between Treynor ratio of securities and Sharpe ratio of securities and positive linear relation ship between natural logarithm of standard deviation of securities' return and square of co-variance between securities' return and market return. These two strategies are portfolio selection and asset allocation strategies using order three asset allocation model and general order three opportunity model.

We would like to test the quality or (validity) of order two zero opportunity cost condition using historical data. We observe from results of this study that securities follow TreynorSharpe regression line. Thus, it is reasonable to construct a portfolio that follows Treynor-Sharpe regression line. It follows from fundamental theorem of order two investment opportunity that risk free fundamental portfolio and risky fundamental portfolio are zero opportunity portfolios.

Order three zero opportunity condition suggests that there is linear relation ship between variance of security return and covariance of security return with market return. However, we realized that there is positive linear relation ship between natural logarithm of standard deviation of securities' return and square of co-variance between securities' return and market return using historical data of securities' return. We have introduced portfolio weight-variance regression line constraint in $G O O M$. We have included portfolio market line constraint and portfolio beta estimation constraint in GOOM. Note that $G O O M$ needs optimization tool for its optimal solution.

The tangency portfolio is constructed by the help of efficiency condition and tangency portfolio condition. Let consider efficiency condition and order three zero opportunity condition to construct $O A A M$. Order three Sharpe error condition and order three efficiency condition follow from efficiency condition and order three zero opportunity condition. We use $O A A M$ to determine weight of risky portfolio, risk free portfolio and market portfolio in order three complete portfolio.

The two models $O A A M$ and GOOM are favorable for investors who do not want opportunity gain or loss from the three investment scenarios. Tangency portfolio does not con- sider portfolio beta estimation constraint, portfolio market line constraint and weight-variance regression line constraint. Let us discuss on mean variance portfolio, tangency portfolio and order three complete portfolio. Tangency portfolio is a special case of mean variance portfolio with capital market line constraint. Thus, mean variance portfolio may outperform tangency portfolio because tangency portfolio is constrained by capital market line constraint. However, mean variance portfolio has opportunity cost risk. Since tangency portfolio is a special portfolio with special case of order three opportunity cost condition, it has order three opportunity cost risk. Order three complete portfolio has no order three opportunity cost risk. Therefore, investors who take zero order three opportunity cost would choose order three complete portfolio for their investment portfolio selection and asset allocation decision. In summary, we have relaxed tangency portfolio condition to order three zero opportunity cost condition to construct order three complete portfolio. In summary, the two models $O A A M$ and $G O O M$ are realistic models than mean variance portfolio and tangency portfolio for investors who takes order three zero opportunity cost risk.

\section{Conclusion and recommendation}

In this research, we have introduced basic portfolio conditions, namely, efficiency condition, Sharpe ratio error condition and order three zero opportunity condition. We have proved some mathematical facts related efficiency condition, Sharpe ratio error condition and order three zero opportunity condition. We have shown that there is positive linear correlation between Treynor ratio and Sharpe ratio of companies using empirical study. Furthermore, we observed that logarithm of standard deviation of companies and square of co-variance of companies with market index are positively correlated. We constructed GOOM using portfolio Treynor-Sharpe line and order three opportunity condition. Moreover, we constructed $O A A M$ using efficiency condition, Sharpe ratio error condition and order three zero opportunity condition. Finally, we applied $G O O M$ and $O A A M$ to construct complete portfolio $C P$.

Let us consider practical application of GOOM and $O A A M$. First, we checked Treynor-Sharpe regression model using historical data of securities' return. Second, we checked the validity of weight-variance regression model using historical data of securities' return. Third, we solved for optimal solution of GOOM. We constructed $O T H$ from GOOM. Fourth, we applied $O A A M$ to determine weights of assets in order three complete portfolio. Finally, we evaluated the performance of $O T H$ and $C P$ using Sharpe ratio process. The result of this study shows that $C P$ is outperforming $O T H$ with respect to Sharpe ratio process. Moreover, we constructed $C P$ using proposed models and historical data. The result of this study suggests that investors who want to take order three zero opportunity cost risk should allocate $\frac{2}{3}$ of the investment capital for risky portfolio.

In mean variance model, weight of each company is assumed as independent of time. In this research, we assumed 
that weight of each company is assumed as independent of time. However, in reality weight may be dynamic with respect to time. Therefore, we recommend researchers to study the impact of dynamic (time dependent) weight on portfolio selection. Another research recommendation for researchers is that the study of impact of market index on portfolio selection and asset allocation. This study does not include the impact of economic and fundamental factors on portfolio selection. Moreover, we will study the impact of market factor and dynamic weight in our future study.

\section{REFERENCES}

[1] Virlics, Agnes, Investment decision making and risk, Procedia Economics and Finance, Elsevier, vol. 6, pp. $169-$ 177, 2013, doi: 10.1016/S2212-5671(13)00129-9.

[2] Hue, B and Jinks, A and Spain, J and Bora, M and Siew, S, Investment risk for long-term investors: risk measurement approaches: Considerations for pension funds and insurers, British Actuarial Journal, Multidisciplinary Digital Cambridge University Press, vol. 24, 2019, doi.org/10.1017/S1357321719000102.

[3] Selmier II, W Travis and Penikas, Henry and Vasilyeva, Ksenia, Financial risk as a good, Procedia Computer Science, Elsevier, vol. 31, 2014, pp. 115-123, doi.org/10.1016/j.procs.2014.05.251.

[4] Khanal, Aditya R and Mishra, Ashok $\mathrm{K}$ and $\mathrm{Ku}-$ mar, Anjani, Risk Preference and Adoption of Risk Management Strategies: Evidence from High-Value Crop Production in Emerging Economy, 2019 Annual Meeting, July 21-23, Atlanta, Georgia 291285, Agricultural and Applied Economics Association, 2019, doi.org/10.22004/ag.econ.291285.

[5] Sulaiman, Ebrahim Kunju, An empirical analysis of $f$ nancial risk tolerance and demographic features of individual investors, Procedia Economics and Finance, vol. 2, 2012, pp. 109-115, doi.org/10.1016/S22125671(12)00070-6.

[6] Sulewski, Piotr and Wkas, Adam and Kobus, Pawel and Pogodzinska, Kinga and Szymanska, Magdalena and Sosulski, Tomasz, Farmers' Attitudes towards Risk-An Empirical Study from Poland, Agronomy, Multidisciplinary Digital Publishing Institute, vol. 10, 2020, pp. 1555, doi.org/10.3390/agronomy10101555.

[7] Sutejo, Bertha Silvia and Pranata, Yohanes Kokoh Natan and Mahadwartha, Putu Anom, Demography factors, financial risk tolerance, and retail investors, 15th International Symposium on Management,2018, doi.org/10.2991/insyma-18.2018.20.

[8] Irandoust, Manuchehr, Factors associated with financial risk tolerance based on proportional odds model:
Evidence from Sweden, Journal of Financial Counseling and Planning, Springer, vol. 28, 2017, pp. 155-164, doi.org/10.1891/1052-3073.28.1.155.

[9] Duan, Yaoyao Clare, A multi-objective approach to portfolio optimization, Rose-Hulman Undergraduate Mathematics Journal, vol. 8, no. 1, pp. 12, 2007, https://scholar.rose-hulman.edu/rhumj/vol8/iss1/12.

[10] Nguyen, Hoang, Portfolio Optimization Methods: The Mean-Variance Approach and the Bayesian Approach, The University of Mississippi, 2019, http://thesis.honors.olemiss.edu/id/eprint/1398.

[11] Kresta, Aleš and others, Application of Performance Ratios in Portfolio Optimization, Acta Universitatis Agriculturae et Silviculturae Mendelianae Brunensis, Mendel University Press, vol. 63, pp. 1969-1977, 2015, doi.org/10.11118/201563061969.

[12] Petronio, Filomena and Lando, Tommaso and Biglova, Almira and Ortobelli, Sergio, Optimal portfolio performance with exchange traded funds, VŠB (Technical University of Ostrava, Faculty of Economics), 2014, doi.org/10.7327/cerei.2014.03.01.

[13] Choueifaty, Yves and Coignard, Yves, Toward maximum diversification, The Journal of Portfolio Management, Institutional Investor Journals Umbrella, vol. 35, no. 1, pp. 40-51, 2008, doi.org/10.3905/JPM.2008.35.1.40.

[14] Lee, Ming-Chang and Su, Li-Er, Capital market line based on efficient frontier of portfolio with borrowing and lending rate, Universal Journal of Accounting and Finance, vol. 2, no. 4, pp. 69-76, 2014, doi.org/ 10.13189/ ujaf.2014.020401.

[15] Jiang, Chonghui and Ma, Yongkai and An, Yunbi, The Mean-Variance Model Revisited with a Cash Account, Scientific Research Publishing, 2012, doi.org/ 10.4236/jmf.2012.21006.

[16] Rambaud, Salvador Cruz and Pérez, José García and Granero, Miguel Ángel Sánchez and Segovia, Juan Evangelista Trinidad, Markowitz's model with Euclidean vector spaces, European Journal of Operational Research, Elsevier, vol. 196, no. 3, pp. 1245-1248, 2009, doi.org/ 10.1016/j.ejor.2008.04.021.

[17] Muhinyuza, Stanislas and Bodnar, Taras and Lindholm, Mathias, A test on the location of the tangency portfolio on the set of feasible portfolios, Applied Mathematics and Computation, Elsevier, vol. 386, pp. 125519, 2020, doi.org/10.1016/j.amc.2020.125519.

[18] Centeno, V and Georgiev, IR and Mihova, V and Pavlov, $\mathrm{V}$, Price forecasting and risk portfolio optimization, AIP Conference Proceedings, AIP Publishing LLC, vol. 2164, no. 1, pp. 060006, 2019, doi.org/ 10.1063/1.5130808. 
[19] Odobašić, Sandra and Tolušić, Marija and Tolušić, Zrinka, The application of the CAPM model on selected shares on the Croatian capital market, Ekonomski vjesnik: Review of Contemporary Entrepreneurship, Business, and Economic Issues, Sveučilište JJ Strossmayera u Osijeku, Ekonomski fakultet u Osijeku, vol. 27, no. 2, pp. 297-311, 2014, Corpus ID: 150446856.
[20] Katsikis, Vasilios $\mathrm{N}$ and Mourtas, Spyridon D, Binary Beetle Antennae Search Algorithm for Tangency Portfolio Diversification, Journal of Modeling and Optimization, vol. 13, no. 1, pp. 44-50, 2021, doi.org/10.32732/jmo.2021.13.1.44. 